\title{
Objeto y método: ¿criterios epistemológicos o coartadas para la supervivencia académica?
}

\author{
Francisco Sánchez Pérez
}

Universidad Complutense de Madrid. Facultad de Ciencias Políticas y Sociología frsanche@cps.ucm.es

\section{Resumen}

Este artículo cuestiona los criterios de demarcación que tradicionalmente han sustentado la autonomía disciplinaria entre la sociología y la antropología. Revisa los principales argumentos canónicos que se han venido empleando para justificar la especificidad de ambos campos disciplinarios y plantea la dificultad de sustentar dicha especificidad desde un punto de vista epistemológico. Finalmente, sostiene que la separación disiciplinaria sólo se justifica desde planteamientos de carácter histórico y sociológico.

Palabras clave: epistemología, metodología, criterios de demarcación, identidad disciplinaria, objeto de estudio.

Abstract. Objet and method: epistemological criteria or strategies for the academic survival?

This article puts into question the demarcation criteria that traditionally have sustained the arguments in favour of the disciplinal autonomy between Sociology and Anthropology. In comparing different objects of study and the specific methods used in each of these subjects, the author concludes that the autonomy of both disciplines can only be justified on historical and sociological grounds.

Key words: epistemology, methodology, demarcation criteria, disciplinal identity, object of study.

\section{Sumario}

Introducción La coartada del sujeto

El objeto como criterio Bibliografía

El método como criterio 


\section{Introducción}

El problema de la demarcación entre la antropología y la sociología se empezó a plantear en el momento en que comenzó el proceso de su institucionalización a finales del siglo diecinueve. Desde que Marcel Mauss pretendiera la inclusión de la sociología en una antropología considerada por él como una ciencia general del ser humano, pasando por las reflexiones del grupo de trabajo de Sociología y Antropología celebrado en el Congreso Mundial de Sociología de 1959 en Chicago, hasta nuestros días, no se ha conseguido establecer con meridiana claridad dónde radica la especificidad exclusiva de las dos materias. Antes al contrario, se podría concluir que la línea de separación se ha ido haciendo cada vez más difusa, si no confusa. Lo cual no deja de ser, cuanto menos, paradójico, pues esa incierta línea de demarcación interdisciplinaria ha ido paralela a una paulatina consolidación de los muros de separación institucional (administrativos, burocráticos, académicos), como se hace patente en la gran proliferación de departamentos, revistas especializadas, publicaciones, titulaciones y egresados en una y otra materia. Sin duda, las razones que han conducido a semejante paradoja son complejas y adquieren dimensiones de carácter ontológico y epistemológico; pero también histórico y social, siendo en esta última dirección donde, a mi entender, hay que buscar los argumentos que justifican la existencia autónoma los dos campos académicos. Veamos.

\section{El objeto como criterio}

Desde los años setenta, se ha producido una ingente literatura relativa al papel que tuvo el orden colonial en la génesis de la sociología y la antropología como campos independientes. Fue en aquel contexto geopolítico cuando se establecieron sus respectivos regímenes de competencias disciplinarias, siéndole asignada a la primera la esfera metropolitana y a la segunda, la colonial. Dos ámbitos empíricos cuya transformación en objetos de conocimiento diferenciados responde a la lógica evolucionista que establecía dicotomías tales como: salvaje / civilizado, primitivo / evolucionado, simple / complejo, sin historia / con historia, sin escritura / con escritura. Dicotomías que, no obstante, una vez refutados los fundamentos teóricos del evolucionismo y disuelto el modelo colonial decimonónico, revelaron su futilidad para definir los objetos de estudio de la sociología y la antropología. Se volvieron completamente inoperantes, hasta el punto de acabar por obligar a sociólogos y antropólogos a entrecomillar con frecuencia los otrora evidentes conceptos definitorios de identidad disciplinaria. Más allá de lo anecdótico, ese gesto de rascar el aire con cuatro dedos revela las dificultades que subsisten para adaptar el viejo modelo de demarcación, su léxico, sus conceptos, a un contexto geopolítico distinto. Bien es verdad que ningún antropólogo o sociólogo que se precie se atrevería hoy en día a utilizar semejantes criterios para justificar la existencia de las dos disciplinas; pero lo cierto es que, si bien de un modo más latente que explícito, casi 
más instintivo y atávico que racional, dichas dicotomías se mantienen aún vigentes en el imaginario colectivo a la hora de dar por sentada la particularidad de una materia frente a la otra. Así, a la antropología se la sigue identificando demasiadas veces con aspectos exóticos o marginales respecto de aquello que estudia la sociología, como se pone en evidencia en el uso - que a estas alturas deviene ya en pantomima - de sus respectivas iconografías de identidad (industrias, urbes, tecnología sofisticada, individuos de raza blanca, etc., frente a asentamientos tribales, escenografías rituales o indígenas).

Se objetará a esto que semejante criterio de demarcación responde a tópicos que perviven por inercia, y que, si bien en su momento tuvieron una incidencia capital en la separación de los campos sociológico y antropológico, no son epistemológicamente relevantes. Y se afirmará que lo que en realidad define el objeto de la sociología es que ésta centra su atención en la dimensión social del ser humano, en tanto que el objeto de la antropología se interesa por su dimensión cultural.

Bien, si ha habido un asunto complicado y obtuso de dilucidar en el ámbito teórico de las ciencias sociales, ése es —y sigue siéndolo— la distinción de los conceptos de sociedad y cultura, como dan fe las innumerables disquisiciones encaminadas a intentar su definición. Quedan para los anales de las ciencias sociales las tempranas y persistentes discusiones entre parsonianos y boasianos, justificando los primeros la preeminencia de la acción social sobre los valores culturales, sobre los símbolos, y justamente lo contrario, los segundos. Como ejemplo concreto, valga recordar el artículo que Parsons y Kroeber, "The concep of cultures and social system», publicaron en American Sociological Review. El mismo Alfred Kroeber describió los conceptos de sociedad y cultura como dos caras de una misma hoja de papel carbón. Claude Lévi-Strauss, a su vez, los consideró como dos puntos de vista de una misma realidad.

Con todo, a pesar de las innumerables, sutiles y sofisticadas disecciones que se han practicado para escindir lo social de lo cultural, el problema sigue sin estar resuelto, acaso porque sea irresoluble. En la literatura teórica de las dos disciplinas abunda una inmensa gama de definiciones que se han ensayado de uno y otro concepto en sucesivos intentos de fijar sus peculiaridades teóricas de modo más o menos excluyente: desde las propuestas por las corrientes materialistas a las simbolistas, pasando por las funcionalistas, estructuralistas, estructural-funcionalistas, marxistas, fenomenológicas, etnometodológicas, interaccionistas simbólicas, críticas, hermenéuticas, constructivistas, etc. Todas ellas han ocupado algún momento de la historia de las dos disciplinas y todas han ensayado, directa o indirectamente, su propia definición de objeto, y todas han sido objeto de su correspondiente réplica, acompañada de una definición alternativa desde el campo contrario.

Dirán los más resolutos que el problema de demarcación teórica no debería suponer un obstáculo insalvable; al fin y al cabo, esa misma dificultad de establecimiento de límites también la tienen las mejores familias del colectivo científico, incluida la física. De modo que se puede echar mano al sentido pragmático y, con él, a las propiedades heurísticas que ofrecen las nociones de 
sociedad y cultura para indagar sobre el ser humano. Así, la sociología se especializa en las estructuras, la antropología, en los símbolos; aquélla, en las relaciones sociales, ésta, en los valores, en los ritos, en los mitos. Bien, en gran medida, ése ha sido uno de los modelos más recurrentes para sustentar el statu quo interdisciplinario. El problema es que, más allá de la innegable validez heurística que pueda aportar el discurrir sobre la dimensión social y la dimensión cultural del ser humano, lo cierto es que semejante dicotomía no ha servido para establecer criterios de identidad disciplinaria suficientemente nítidos. Prueba de ello es que tanto en una como en otra materia coexisten no ya planteamientos «sociologistas» $\mathrm{y}$ "culturalistas», sino también, para enrevesar aún más el asunto, planteamientos sociologistas de contenido más culturalista que incluso algunos culturalistas mismos, y viceversa.

Entonces, si el criterio geopolítico evolucionista no es apropiado para establecer las líneas de demarcación entre estas dos ciencias sociales, ni tampoco los conceptos teóricos de sociedad y cultura son operativos para justificar la existencia de dos campos disciplinarios con identidad propia, habida cuenta de que ambos inspiran indistintamente tanto a sociólogos como a antropólogos, ¿qué criterios quedan para definir sus respectivos objetos de estudio?: ¿la noción de «distancia» esgrimida por Lévi-Strauss?, ¿la de «alteridad», sostenida todavía por William Y. Adams en su libro Las raíces filosóficas de la antropología?

Qué duda cabe que estas dos nociones — distancia y alteridad - también tuvieron en su momento un papel decisivo para justificar la autonomía de campo de la sociología y la antropología. Mientras que el objeto de estudio de la primera quedaba definido por rasgos de proximidad y afinidad respecto de los sujetos de conocimiento, el de la segunda lo estaba en función de la distancia y la diversidad. Así, el prójimo y el ajeno, nosotros y ellos, constituían categorías esenciales con la suficiente entidad para definir, por sí mismas, la naturaleza ontológica de dos objetos de estudio distintos. ¿Cómo se iban a cuestionar las diferencias existentes entre un nativo trobriandés y un antropólogo británico, entre un indio kwakiutl y un obrero londinense, o la afinidad entre un sociólogo neoyorquino y un habitante del barrio del Bronx neoyorquino? ¿'No eran acaso suficientes para conformar por sí mismas objetos de conocimiento diferenciados? Y lo fueron, en efecto, pero sólo hasta las postrimerías de la Segunda Guerra Mundial. Con la independencia de los países del Tercer Mundo y la incorporación a la esfera de las ciencias sociales de colectivos de profesionales pertenecientes a ámbitos considerados como periféricos, la sociología empieza a interesarse por problemas del Tercer Mundo derivados del incipiente proceso de globalización; la antropología, a su vez, vuelve su mirada al Primer Mundo. Progresivamente, las nociones de distancia y proximidad se han revelado como un mero referente geográfico fuertemente cargado de etnocentrismo, a todas luces irrelevante desde un punto de vista epistemológico como argumento para distinguir y justificar sendos objetos de estudio. En cuanto a la tesis de la alteridad empleada por Adams, quizás debería bastar para rebatirla la reflexión con la que Tzvetan Todorov inicia su libro 
La conqête de l'Amerique. La question de l'autre (1982): "On peut découvrir les autres en soi, se rendre compte de ce qu'on n'est pas une substance homogène, et radicalement étrangère à tout ce qui n'est pas soi: je est un autre».

De modo que si no se ha resuelto la distinción teórica de los objetos de la sociología y la antropología, pero, sobre todo, si en la práctica no hay modo de diferenciar lo que investigan sociólogos y antropólogos, ¿qué argumento epistemológico queda para justificar la pretendida autonomía disciplinaria: el método?

\section{El método como criterio}

Uno de los asuntos sustanciales a la hora de tratar los fundamentos de demarcación disciplinaria se refiere a la relación que guarda el método con el objeto de estudio. Al respecto, hay quienes, siguiendo la distinción de Windelband entre ciencias nomotéticas — generadoras de leyes-y ciencias idiográficas — centradas en los fenómenos particulares - concuerdan en afirmar que el método no depende del objeto de estudio disciplinario, sino del interés cognoscitivo. Algo que queda bien reflejado en alguien tan significativo para el asunto que aquí nos ocupa como fue Emile Durkheim, y la decisiva influencia que, en contra de lo que él mismo pensaba al respecto, tuvo su obra en la bifurcación de los derroteros que tomaron la sociología y la antropología. No deja de resultar revelador el modo en que se apropiaron de este autor y de su obra los antropólogos y los sociólogos, hasta el punto de considerarlo, cada uno por su lado, como uno de sus tótemes de identidad disciplinaria. Sólo que un tótem bifronte, del que cada disciplina adoptó el perfil que mejor se acoplaba a sus respectivos objetos de interés, lo cual implicaba la asimilación de los modelos teóricos con los que Durkheim concibió sus trabajos, mas también los métodos empleados en los mismos. Así, mientras que la sociología se apropiaba del análisis estadístico aplicado en El suicidio, la antropología incorporaba a su bagage metodológico la utilizada en Las formas elementales de la vida religiosa, concretamente la observación, bien que observación indirecta, toda vez que Durkheim se sirvió de testimonios de misioneros y gestores coloniales. A partir de aquí, basta un somero rastreo de la influencia que ejerció el sociólogo francés sobre la antropología y la sociología para entender algunas de las razones que llevaron a la primera a adoptar la observación participante y a la segunda, el muestreo estadístico como principales procedimientos para la investigación.

El hilo conductor de la versión antropológica nos lleva directamente a Radcliffe Brown, el introductor de la teoría durkheimiana en la antropología británica. Por su lado, Malinowski, con su defensa del trabajo de campo in situ de larga duración, dota al método etnográfico de un cariz paradigmático como método de la antropología. Al otro lado del atlántico, Franz Boas lo dota de una mayor densidad teórica con su particularismo histórico. En adelante, la etnografía será considerada no ya el método por excelencia de la antropología, sino que llegará incluso a ser confundida con ella, como de hecho ocurre cuan- 
do Clifford Geertz afirma (1988, p. 20): «En antropología, o en todo caso en antropología social, lo que hacen los que la practican es etnografía. Y comprendiendo lo que es la etnografía o más exactamente lo que es hacer etnografía se puede comenzar a captar a qué equivale el análisis antropológico como forma de conocimiento".

Por su lado, en el campo de la sociología adquieren una especial relevancia, por la enorme influencia que tienen, los trabajos señeros de dos teóricos de la Universidad de Columbia: Robert K. Merton y Paul F. Lazarsfeld. Concretamente, la obra de éste último fue decisiva para la instauración de una sociología de marcado carácter empirista. Sus trabajo The Peoplés Choice: How the Voters Makes Up His Mind in a Presidential Compaing (1944), hecho en colaboración con Berelson y Gaudet, es considerado pionero de una larga serie de investigaciones realizadas mediante la aplicación de cuestionarios que habrían de imponerse en la práctica sociológica desde mediados del siglo XX. Como dice J. Picó (2003, p. 134), «Lazarsfeld asentó en Columbia, modificándola, la tradición inglesa del social survey; una análisis esencialmente causal que se propone manejar datos no experimentales después de ser recogidos a través de la cuantificación y el juego combinatorio de las variables».

No siempre, sin embargo, se acepta esta manera de entender el papel que desempeña el método en tanto que factor definitorio de las competencias disciplinarias. Como tampoco se acepta la adscripción del procedimiento inductivo, micro, cualitativo e idiográfico a la antropología, y el análisis deductivo, macro, cuantitativo y nomotético a la sociología. Fundamentalmente, porque se niega que el método pueda ser concebido separado de la investigación en que se lo emplea. Como decía Augusto Comte y nos recuerdan Bourdieu, Chamboredom y Passeron (1989, p. 10): «El método no es susceptible de ser estudiado separadamente de las investigaciones en que se lo emplea», lo que viene a significar que el método ha de contar en su misma concepción y diseño con las propiedades del objeto, es decir, que ha de mantener algún tipo de correspondencia isomorfa con lo investigado.

Claro que mantener esto último equivale, de alguna manera, a rechazar la tesis de la autonomía entre la sociología y la antropología, como así hace Bourdieu — sin duda uno de los máximos exponentes del asunto que aquí nos ocupa-, cuando declara que todo su trabajo durante veinte años buscaba abolir la oposición entre la etnología y la sociología. Difícil empeño el suyo, cuando para ello hay que enfrentarse, no ya con complicados artificios teóricos o metodológicos que apuntan en la dirección opuesta, sino también con el enorme peso de la inercia institucional, empecinada en seguir añadiendo ladrillos al muro de separación disciplinaria. Un muro cuya inconsistencia epistemológica se pone de manifiesto a poco que hagamos un somero repaso de la historia de la metodología en estas dos ciencias sociales.

En lo que al campo de la sociología se refiere, ya desde los tempranos años veinte se empezó a practicar sistemáticamente una metodología etnográfica en el seno de la Escuela de Chicago. Así lo evidencian trabajos como: The Gang (1927), de Thrasher; The Gold Coast an the Slum (1929), de Zorbaugh; The 
Polish Peasant in Europe and America, 1918-1920, de Thomas y Znanieki; Yankee City (1941-1959), de Lloyd Warner, o Street Corner Society (1943), de Whyte. Todos ellos son producto de investigaciones realizadas mediante observación participante, entrevistas en profundidad y documentos personales. Es decir, la metodología propuesta por el fundador de la Escuela, Robert Park (1952, p. 15), quien argumenta: «Los mismos y pacientes métodos de observación que los antropólogos, como Boas y Lowie, habían empleado para estudiar la vida y las costumbres de los indios norteamericanos podrían ser utilizados —incluso con más provecho- para investigar las creencias, prácticas sociales e ideas generales de la vida vigentes en Little Italy, en el Lower North Side de Chicago». Más tarde, a finales de los años sesenta, los sociólogos Glasser y Strauss publican The Discovery of Grounded Theory: Strategies for Qualitative Research. El primero de ellos es seguidor del interaccionismo simbólico y el segundo, discípulo de Lazarsfeld en la Universidad de Columbia. La Grounded Theory supone un planteamiento que ha tenido una gran repercusión en el ámbito de la metodología sociológica, que intenta conjugar los modelos estadísticos inspirados en el positivismo científico-social con la metodología cualitativa.

En cuanto al marco de la antropología, Marvin Harris, en su esfuerzo por fundamentar su concepción materialista de la cultura, critica la excesiva influencia del particularismo histórico de Boas en la disciplina echando mano de la siguiente cita de Kroeber, escrita en la recensión que éste hizo del libro de R. Lowie Primitive Society: (1978, p. 524) «Mientras sigamos ofreciendo al mundo sólo reconstrucciones de detalles concretos y mostremos una actitud uniformemente negativa frente a las conclusiones de más alcance, el mundo mostrará poco interés por la antropología». Y, en este mismo sentido, Harris añade (1978, p. 525): «Murdock no sólo ha rechazado las críticas de Boas al método comparativo, sino que ha llegado más lejos que ninguna de las otras figuras de la restauración nomotética en el uso de técnicas estadísticas para hacer generalizaciones que puedan ser sometidas a verificación». Además, Harris resalta la conexión que dicho autor hace entre la etnografía histórica, la estadística moderna y el método de estudio estadístico comparativo intercultural, e incluso va aún más lejos cuando afirma (1978, p. 530): «Los Human Relations Area Files», creados en 1937 por Murdock, «representan un logro culminante de una línea ininterrumpida de entusiastas de la representación estadística que desde Murdock se remonta al mismo Taylor, de cuyo ensayo On a method of investigating the development of institutions: applied to laws of marriage an descent partió el impulso originario». Finalmente, el mismo Harris declara (1978, p. 563): «las más fecundas generalizaciones sobre la historia se han de encontrar estudiando la relación entre los aspectos cuantitativos y cualitativos de los otros dominios de fenómenos socioculturales, que son la variable dependiente».

Bien, no creo que sea necesario seguir ampliando un panorama que, lo miremos por donde lo miremos, sea desde la perspectiva teórica o de las prácticas, niega la especificidad metodológica de nuestras dos disciplinas. Así que, 
o concluimos que nos encontramos ante un masivo ejercicio de intrusismo profesional, de un juego de falsas identidades, o habremos de empezar a pensar que los principios sobre los que se sustenta la autonomía de los campos antropológico y sociológico no responden a razones de carácter epistemológico, como así pretende el cánon académico. Pues, si las dicotomías de orden teórico sociedad/cultura, civilizado/primitivo, nosotros/ellos, etc. no justifican la razón de ser de dos disciplinas; ni tampoco las dicotomías de orden metodológico cualitativo/cuantitativo, inductivo/deductivo, nomotético/idiográfico, macro/micro, etc. ¿qué justifica la supervivencia de las dos disciplinas en el campo de las relaciones académicas?

\section{La coartada del sujeto}

La cuestión es si la existencia de dos sujetos de conocimiento con un objeto y una metodología compartidos, puede ser considerada como criterio epistemológico de demarcación disciplinaria. Pierre Bourdieu, ya lo hemos leído, dice que no. Y con él numerosos sociólogos y antropólogos que, haciendo caso omiso de los preceptos canónicos de sus respectivas disciplinas, echan mano de autores, teorías y metodologías de uno u otro campo para construir e investigar sus objetos de estudio. Lo cierto es que cada vez resulta más difícil encontrar rasgos distintivos entre lo que hacen los sociólogos y lo que hacen los antropólogos; y cuando ello es posible, se explican mejor a la luz de los viejos modelos que apelando a razones de orden epistemológico. Significativamente, esos rasgos distintivos adquieren su máxima expresión en manuales de introducción, memorias de oposiciones o congresos profesionales, donde se ritualizan los referentes de identidad disciplinaria. Además, subyacen de modo más o menos subrepticio en los temas que se abordan, disimulados en los léxicos, camuflados en las retóricas. Retóricas que contribuyen a dotar a la sociología y a la antropología de una identidad disciplinaria aparente, de la que se vale el stablishment académico para argumentar la autonomía de los dos campos.

Habrá quienes piensen que, aún compartiendo objeto y método, la coexistencia de los dos colectivos académicamente constituidos como sujetos de conocimiento no ha sido un inconveniente; por el contrario, ha supuesto un enriquecimiento mutuo y ha procurado grandes aportaciones al conocimiento científico-social general. Habrá también quienes, aún confundiendo validez epistemológica con solidez institucional, justifiquen el mantenimiento del statu quo argumentando el potencial heurístico que comporta la presencia de dos «sensibilidades», dos "culturas», dos «thought communities», en el sentido que confiere a esta noción el Programa Fuerte de la sociología del conocimiento. Y habrá quienes, haciendo suya la fórmula que empleara Lévi-Strauss para referirse a los mitos, justifiquen el statu quo disciplinario diciendo que las dos disciplinas son «buenas para pensar». En otras palabras: que se dejen las cosas como están.

Va de suyo que semejante forma de plantear el problema de la demarcación choca de lleno con los preceptos de la más elemental lógica científica. 
Al fin y al cabo, como predicaba Feyerabend, también el budú es bueno para pensar, y no por ello tiene cabida en el ámbito científico, si no es como objeto mismo de interés. De manera que a ningún aspirante a sociólogo o antropólogo se le ocurrirá defender formalmente como criterio de demarcación entre la antropología y la sociología la existencia de dos colectivos académicos, si no quiere verse condenado al ostracismo profesional. Más le valdrá no empeñarse en cuestionar los fundamentos canónicos de demarcación y dedicarse a emplear argumentos, si no epistemológicamente válidos, al menos académicamente correctos, para sustentar sus aspiraciones a verse reconocido en el seno de uno u otro colectivo.

El problema es que ese «dejar las cosas como están» no sólo es incoherente con el prurito científico que se le supone a cada disciplina, sino que puede comportar más problemas que ventajas. Para empezar, presupone un planteamiento lastrado en la lógica positivista decimonónica, pues sobreentiende que las disciplinas de conocimiento son dispositivos neutros de captación de una realidad dada, independiente del sujeto que la experimenta. Equivale a pensar algo así como que el mundo real está parcelado y a cada parcela le corresponde un orden disciplinario ad hoc. Sólo que la cosa no funciona así, toda vez que en el modo como se realiza esa parcelación tiene mucho que ver la acción determinante de los sujetos que la efectúan. Y además, los dispositivos teóricos y metodológicos de los que se valen para ello no son tan inocentes como pretenden quienes quieren dejar las cosas como están. Las teorías y los métodos, como también las condiciones sociales en las que el sujeto los produce y aplica, contribuyen a construir el modo como se parcela la realidad que se pretende conocer. De ahí la necesidad de efectuar el ejercicio de permanente vigilancia epistemológica que propugnara Gastón Bachelard sobre la acción cognoscitiva del sujeto; de ahí la necesidad de ejercer esa misma vigilancia sobre las condiciones sociales en que se efectúa dicha acción, como plantea Bourdieu. Una vigilancia que, en lo que aquí nos concierne, permita detectar y, llegado el caso, corregir los efectos negativos que puedan comportar el mantenimiento de un statu quo académico.

Un buen ejemplo del efecto negativo lo tenemos en esa suerte de «tribalización» a la que los antropólogos tantas veces han sometido a su objeto, con tal de que se ajustara al criterio de demarcación establecido por el canon heredero del evolucionismo. Consecuencia de ello ha sido la debilidad que siempre ha tenido la antropología para abordar problemas empíricos no apropiados para el alcance metodológico de la observación participante. Justamente lo contrario de lo que le ha sucedido a la sociología con su genuina técnica del muestreo respecto de estudios de alcance micro y cualitativo. Como dicen Bourdieu y otros (1994, p. 129-130): «Más que el sociólogo, amenazado más bien por una distancia respecto de su objeto que no siempre es distanciamiento epistemológico, el etnólogo, como todos los que recurren a la observación participante, corre el peligro de tomar el "contacto humano" por un medio de conocimiento $y$, sensible a las exigencias y seducciones de su objeto que traicionan las evocaciones nostálgicas de lugares y gentes, debe realizar un esfuerzo particular 
para construir una problemática capaz de romper las configuraciones singulares que le proponen los objetos concretos».

Es verdad que lo dicho es más una crítica a las implicaciones epistemológicas del uso restringido de tal o cual técnica de investigación que a la totalidad de las disciplinas. Al fin y al cabo, éstas pueden evitar los efectos negativos que se derivan de ello. Y, de hecho, así lo hacen, como ya se ha mostrado en el apartado anterior, bien que de un modo a todas luces desequilibrado, siendo puramente testimonial el empleo de la metodología idiográfica en la sociología, y prácticamente inexistente la nomotética en la antropología que últimamente se hace. Con todo, no es este desequilibrio el que aquí se critica - que no es sino consecuencia directa de la especialización disciplinaria basada en los viejos modelos epistemológicos-, sino el hecho de que se plantee el mismo problema metodológico en el seno de las dos disciplinas, sin que ello llegue a afectar en lo más mínimo a sus respectivos estatutos de autonomía. Uno termina por preguntarse cómo es posible que prolifere tanto discurso sobre la identidad disciplinaria de la sociología, por un lado, y de la antropología, por otro, sus crisis, sus dificultades para definir las fronteras, sus objetos de estudio, sus métodos, sin prestar atención al problema de demarcación interdisciplinaria. De hecho, sorprende la frecuencia de discusiones habidas al respecto en el pasado, cuando todo parecía estar más claro en el panorama disciplinario, y lo poco o nada que se hace en la actualidad. ¿Acaso porque, siendo ya evidente la futilidad de los argumentos de demarcación no queda sino mantener las apariencias?

Es ante semejante panorama cuando uno empieza a barruntar si la manifiesta dejadez para revisar los criterios de demarcación interdisciplinaria no responde a otra cosa que a un subrepticio interés del stablishment profesional de la sociología y la antropología en mantener las cosas como están; cuando surge la sospecha de que los discursos encaminados a sustentar la especificidad de sus respectivos objetos y métodos no son más que coartadas retóricas para justificar la existencia de los dos colectivos profesionales y asegurar su reproducción como colectivo en el campo de relaciones académicas, un campo en el que parece reinar una suerte de pacto tácito de no cuestionamiento mutuo, que admite incluso la posibilidad de solapamiento de materias y hasta la usurpación de competencias disciplinarias, con tal de que no se ponga en riesgo la trama de intereses que sustenta el orden académico establecido. Un pacto que, si bien permanece latente en períodos de normalidad, entra en crisis en momentos de cambio, cuando surge el riesgo de ver conculcado el capital académico de los profesionales de uno y otro campo. Es entonces cuando se echa mano de las coartadas del objeto y del método para que las cosas sigan como están, incluso por quienes se dedican a cuestionar la validez de los criterios de demarcación en el seno de sus respectivas disciplinas. Después de todo, ¿qué más da llamarse antropólogos o sociólogos, si al fin y al cabo hacemos lo mismo?

Lo mismo no, dirán quienes se empecinan en mantener inhiestos unos muros de separación hechos no con materiales epistemológicos, sino con una amalgama hecha de retazos del pasado y elementos burocrático-administrati- 
vos. Son esos antropólogos que creen atenerse a la disciplina que profesan porque han diseñado su objeto de estudio con rasgos étnicos, marginales, grupos pequeños, etc.; porque se interesan por valores, por símbolos o por rituales; o porque, incluso cuando estudian objetos genuinamente sociológicos, lo hacen mediante el método etnográfico. Esos sociólogos convencidos de que no hacen antropología porque aplican la técnica del muestreo para estudiar la inmigración o aplican el análisis multivariable para el análisis de valores, o porque desisten de echar mano de la observación participante, porque «de eso se encargan los antropólogos»; esos antropólogos que nunca han oído hablar de los focus groups. Son quienes hablan del Bourdieu sociólogo cuando se refieren a La distinción y del Bourdieu antropólogo cuando se refieren a sus trabajos de campo en Kabilia. Esos devotos de la ortodoxia disciplinaria que organizan congresos, editan revistas, diseñan planes de estudio, escriben libros y artículos, promueven asociaciones, encuentros, seminarios, establecen equipos y redes de investigación, poniendo especial cuidado en preservar la imagen de marca, el marchamo de denominación de origen. Son quienes, valiéndose de los mecanismos burocrático-administrativos de reproducción académica, tratan de establecer qué es antropología, qué sociología, quién es antropólogo, quién sociólogo. Ésos son los que, de forma tácita o explícita, conscientes o no de las implicaciones que comportan sus actitudes, reproducen el statu quo disciplinario asumiendo los tradicionales criterios de demarcación disciplinaria. Criterios que, cuando llega el caso, son usados como coartadas para su supervivencia académica.

Cómo haya que proceder para superar este estado de cosas es algo que escapa a este artículo, cuyo propósito no es otro que el de llamar la atención sobre un problema al que se le ha dejado de conceder la trascendencia que tiene. Ni qué decir tiene que no se trata de proponer la desaparición de la antropología o la sociología; tampoco la inclusión o la disolución de una de ellas en la otra; ni siquiera un radical e imposible acto de refundación en una nueva disciplina unitaria que dé cuenta de la realidad sociocultural. Se trata de que no se continúen poniendo más ladrillos burocrático-administrativos en los muros de separación; de que se terminen de demoler los que aún permanecen erectos; de contribuir a que se instauren nuevas estructuras académicas que superen los viejos modelos, nuevos planes de estudio, nuevas materias. Estructuras que permitan la constitución de un sujeto de conocimiento que pueda justificar su adscripción disciplinaria sin tener que echar mano de coartadas. Un sujeto que establezca criterios de demarcación epistemológicamente fundados para la definición de su objeto de estudio e imaginar la metodología más pertinente para investigarlo, sin sentirse deudor del formalismo disciplinario. En fin, un sujeto de conocimiento que no conciba el atenerse a los principios de su disciplina como un fin en sí mismo, sino como un medio, un instrumento válido para intentar arrojar luz sobre la condición sociocultural del ser humano. 


\section{Bibliografía}

ADAMS, William Y. (2003). Las raices filosóficas de la antropología. Madrid: Trotta. Alvesson, M.; SkÖldberg, K. (2000). Reflexive Methodology. New Vistas for Qualitative Research. Londres: Sage.

BACHELARD, G. (1972). La formación del espiritu cientifico. Madrid: Siglo XXI.

BARnes, B. (1976). Scientific Knowledge and Social Theory. Londres: Routledge and Kegan Paul.

BloOr, D. (1976). Knowledge and Social Imagery. Londres: Routledge and Kegan Paul. Bourdieu, P. (1984). Homo Academicus. París: Les Éditions du Minuit.

- (1995). Respuestas: por una antropología reflexiva. México: Grijalbo.

- (1996). Cosas dichas. Madrid: Gedisa.

Bourdieu, P.; Chamborendom, J. C.; Passeron, J. C. (1989). El oficio de sociólogo. Madrid: Siglo XXI.

DENZING, N. K.; LinCON, Y. (eds.) (1994). Handbook of qualitative research. California, Sage: Thousand Oaks.

DuRKHEIM, E. (1968). Las formas elementales de la vida religiosa. Buenos Aires: Schapire. - (1982). El suicidio. Madrid: Akal.

- (1964). Las reglas del método sociológico. Buenos Aires: Dédalo.

GEERTZ, C. (1988). La interpretación de las culturas. Barcelona: Gedisa.

Glaser, F. G.; Strauss, A. L. (1967). The Discovery of Grounded Theory: Strategies for Qualitative Research. Chicago: Aldine.

HARRIS, M. (1978). El desarrollo de la teoría antropológica: Una historia de las teorías de la cultura. Madrid: Siglo XXI.

Knorr-Cetina, K; MulKay. M. (eds.) (1983). Science Observed. Londres: Sabe.

Kuper, A. (2001). Cultura. La versión de los antropólogos. Barcelona: Paidós.

Lazarsfeld, P. F.; Berenson; Gaudet, H. (1944). The people choice. Nueva York: Columbia University Press.

LÉVI-STRAUSS, C. (1973). "Le champ de l'anthropologie», en Antropologie Structurale Deux. París: Plon.

LowIE, R. (1972). La sociedad primitiva. Buenos Aires: Amorrortu.

PiCÓ, J. (2003). Los años dorados de la sociología (1945-1975). Madrid: Alianza.

RADCliffe BRown, A. R. (1975). El método de la antropología social. Barcelona: Anagrama.

Thomas, W. I.; Znaniecki, F. (1984). The Polish Peasant in Europe and America, 1918-1920. Urbana: University of Illinois.

Thrasher, F. M. (1927). The Gang. A study of 1313 gangs in Chicago. Chicago: The University of Chicago Press.

Todorov, T. (1982). La conquête de l'Amerique: La question de l'autre. París: Seuil. Whyte, W. F. (1943). Street Corner Society. Chicago: University of Chicago Press. WATZlaWICK, P.; KRIEG, P. (comps.) (1994). El ojo del observador: Contribuciones al constructivismo. Barcelona: Gedisa.

Zorbaugh, H. W. (1929). The Gold Coast an the Slum. Chicago: The University of Chicago Press. 